\title{
Entangled Photon Pairs and Young's Experiment
}

\author{
Lewis Nash \\ Marietta, GA, USA \\ Email: lewis.r.nash@gmail.com
}

How to cite this paper: Nash, L. (2021) Entangled Photon Pairs and Young's Experiment. Journal of Quantum Information Science, 11, 135-142.

https://doi.org/10.4236/jqis.2021.114011

Received: June 17, 2021

Accepted: November 30, 2021

Published: December 3, 2021

Copyright $\odot 2021$ by author(s) and Scientific Research Publishing Inc. This work is licensed under the Creative Commons Attribution International License (CC BY 4.0).

http://creativecommons.org/licenses/by/4.0/

\begin{abstract}
Bohm's variation of the Einstein-Podolsky-Rosen thought experiment may be used to reveal the path a photon travels in Young's experiment without destroying the observed interference pattern. Photons emitted by a light-source with zero spin are incident on a screen with two narrow slits separated by a small distance, with antiparallel polarized photonic spin filters placed in front of both slits. It follows that the slit through which an incident photon passed to form an interference pattern can be determined by performing an intermediate delayed choice measurement. This experimental probe is ideal for penetrating the shroud of mystery surrounding the wave-particle duality exhibited by quantum phenomena.
\end{abstract}

\section{Keywords}

Double-Slit, Entangled, Wave-Particle, Photon Pair, Interference

\section{Introduction}

The physics of the extremely small has confounded physics for little over a century. Quantum mechanics is the mathematical machinery developed in the mid 1920's by physicists such as Werner Heisenberg, Erwin Schrodinger, and Max Born [1] [2] [3] to name a few, to describe the strange and perplexing phenomenon that has come to be known as the wave-particle duality [4] [5] of nature. Richard Feynman, discussing the double-slit experiment in his admirable introduction to quantum mechanics, notes that this wave-particle dual behavior contains the basic mystery of quantum mechanics. In fact, he goes so far as to say: "In reality it contains the only mystery".

Thomas Young's seminal double-slit experiment [6] is one of the most notable experiments that clearly displays the wave-particle duality of nature, which ulti- 
mately helped to shape the fundamental building blocks of quantum mechanics. Thus, in this paper we propose a novel thought experiment that reveals the path that photons take in Young's double-slit apparatus without destroying the observed interference pattern. With the aid of advanced photonic devices such as polarized photonic spin (PPS) filters [7] and a spin photodetector [8], our analysis shows that this experiment can provide empirical proof that the photon passes through one slit or the other and not through both slits simultaneously, without causing the observed interference pattern to vanish. This experimental probe is ideal for penetrating the shroud of mystery surrounding the wave-particle duality exhibited by quantum phenomena.

\section{EPR-Bohm thought Experiment with Photon Pairs}

Albert Einstein, Boris Podolsky, and Nathan Rosen meant to look for an experiment that could measure, indirectly but simultaneously, two mutually exclusive quantities like position and momentum. Such results would contravene the predictions of quantum mechanics, which allows the measurement of only one such quantity at any other time; that is why this particular thought experiment has come to be known as the EPR paradox [9].

In 1952 David Bohm showed that the paradox could be set up not only with continuously varying quantities like position and momentum, but also with discrete quantities like spin. Thus, let us consider the EPR-Bohm thought experiment [10] for photon pairs. Suppose a light source $S$ with zero spin that is at rest spontaneously emits two photons simultaneously. In accordance with the conservation of momentum, the two photons diverge from $S$ in opposite directions, which I have labeled A and B in Figure 1 below. Since the initial total spin angular momentum of the system is zero and must be conserved, then the final total spin angular momentum of the system is zero, as well.

According to quantum mechanics, we can arrange our light source so that each emitted photon pair occupies a quantum state known as a singlet or spin singlet state. The photons of a photon pair are thus said to be entangled (or correlated). This can be viewed as a quantum superposition of two states, which we shall call state $|R\rangle$ and state $|L\rangle$, for photons with right and left-handed spin, respectively. This is a state of entangled spin angular momentum. Because circular polarization is assigned relative to the direction of propagation, the singlet state of the two counter-propagating photons denoted $\gamma_{A}$ and $\gamma_{B}$, respectively includes two right-handed $|R, R\rangle$ and two left-handed $|L, L\rangle$ photons, which

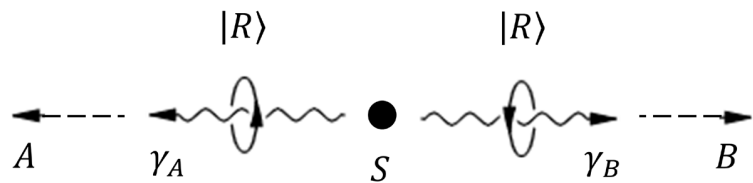

Figure 1. A particle $S$ with zero spin decays into two photons $\left(\gamma_{A}\right.$ and $\left.\gamma_{B}\right)$, traveling in opposite directions $\mathrm{A}$ and $\mathrm{B}$ with right-handed spin $|R\rangle$ which satisfies conservation of total spin angular momentum. 
are states of zero total angular momentum [11].

Let us assume that in state I, $\gamma_{A}$ and $\gamma_{B}$ each have right-handed spin; and in state II, $\gamma_{A}$ and $\gamma_{B}$ each have left-handed spin. Hence, the quantum state occupied by each entangled photon pair emitted by our spin-zero source is described by the following relation

$$
|\Psi\rangle=\frac{1}{\sqrt{2}}[|R, R\rangle+|L, L\rangle],
$$

where $|R, R\rangle$ is the state vector for state I and $|L, L\rangle$ is the state vector for state II. In general, the singlet state is symmetric in the circular polarization basis and exhibits perfectly correlated spin components when locally measured along any axis.

One finds that upon measuring the spin of $\gamma_{A}$. The measurement may give one of two possible outcomes either state $|R\rangle$ or $|L\rangle$. Suppose the measurement gives $|R\rangle$, then informally speaking, the quantum state of the system collapses into state $I$. The quantum state determines the probable outcomes of any measurement performed on the system. Consequently, in this case the measurement of the spin of $\gamma_{B}$ gives $|R\rangle$ with $100 \%$ probability. Similarly, if the measurement of the spin of $\gamma_{A}$ gives $|L\rangle$, then the system collapses into state II and the subsequent measurement of the spin of $\gamma_{B}$ gives $|L\rangle$ with $100 \%$ probability.

\section{Young's Experiment for Single Photons}

The acceptance of the wave character of light was firmly established in 1801, when the English physicist and physician Thomas Young demonstrated optical interference with his now-classic two-slit interference experiment. In Young's experiment, sunlight was passed through a pinhole on a board. The emerging beam fell upon two pin holes, separated by a few millimeters, on a second board. The light emanating from the two pinholes then fell on a screen where a pattern of bright and dark spots was observed [6]. This pattern, called fringes, can only be explained through interference, as a wave phenomenon. Today, aware of the physics, we generally replace the pinholes with narrow slits that let through much more light.

Over one hundred years later in 1909, Sir Geoffrey Ingram Taylor, while an undergraduate, set up Young's experiment and gradually reduced the intensity of the incident light beam to such an extent that there would only be one quantum of energy (a single photon) in the apparatus at any given instant [12]. The resulting interference pattern was recorded using a photographic plate with a very long exposure time. To his disappointment, he found no noticeable change in the pattern, even at the lowest intensities.

At this point one may naturally ask, doesn't it take two waves to interfere? Can a single photon split in half, pass through both slits simultaneously, and then interfere with itself? Quantum mechanics unambiguously says yes. As Paul Dirac, one of the pioneers of relativistic quantum field theory, put it: "Each photon 
interferes only with itself. Interference between different photons never occurs" [13]. The proof that quantum mechanics offers for this absurd proposition is known as the principle of quantum superposition [14]; and has no classical analogue. Quantum superposition is supposedly responsible for all the miraculous magic that quantum systems are capable of, which have been completely verified by a myriad of experiments and modern technologies.

We must not however get carried away and conclude from the interference pattern that photon waves are classical waves, because photons do arrive at the photographic plate in a definite way-one localized flash per photon. It is the totality of spots made by many photons that forms the wave interference phenomena. Analogous to electron waves, photon waves are probability (or relativistic de Broglie) waves [15]. Hence, we say that the probability of a photon arriving at the light areas on the observation screen is high while the probability of a photon arriving at the dark areas is low. Accordingly, the corresponding state of the photons exiting the two slits is represented by the following expression:

$$
|\Psi\rangle=\frac{1}{\sqrt{2}}\left[\left|\psi_{1}\right\rangle+\left|\psi_{2}\right\rangle\right]
$$

where $\left|\psi_{1}\right\rangle / \sqrt{2}$ and $\left|\psi_{2}\right\rangle / \sqrt{2}$ represent the (normalized) probability amplitudes for a photon to pass through either slit 1 or slit 2, respectively.

\section{Young's Experiment with Parallel PPS Filters}

Suppose there exists a device which allows one to filter out photons with a desired spin state from incident light with mixed spin states without disturbing the quantum coherence [16] of their wave functions. We will call such a device a polarized photonic spin (PPS) filter. An ideal PPS filter allows left-handed photons to pass through it without divergence in one direction and right-handed photons to pass through it without divergence in the opposite direction.

Let us imagine that a spin-zero source decays and emits entangled photon pairs [17] with one photon of the entangled pair traveling in direction $\mathrm{A}$ and the other traveling in direction B (see Figure 2 for reference). The photons traveling

Parallel PPS filters

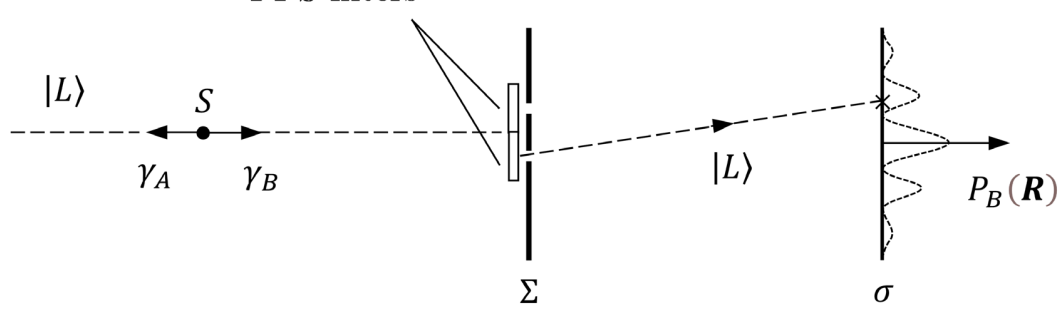

Figure 2. Photon $\gamma_{B}$ of an entangled photon pair, emitted by a spin-zero light source $S$, is incident on a screen $\Sigma$ with two narrow slits covered by two parallel PPS filters. Upon passing through the filters the incident photons with right-handed spin $|R\rangle$ are quickly diverged, while those with left-handed spin $|L\rangle$ are permitted to travel to the observation screen $\sigma$ undisturbed. 
in direction B are incident on a screen with two closely spaced narrow slits and a PPS filter placed in front of each slit, which are oriented to allow only photons with left-handed spin to pass through without divergence. The left-handed photons that pass through the parallel PPS filters are transmitted without disturbing the coherent superposition of $\left|\psi_{1}\right\rangle$ and $\left|\psi_{2}\right\rangle$. The relation for the state of the photons $\gamma_{B}$ exiting the two-slit screen has the perspicuous form

$$
|\Psi\rangle_{B}=\frac{1}{2}\left[\left|\psi_{1}\right\rangle|L, L\rangle+\left|\psi_{2}\right\rangle|L, L\rangle+\left|\psi_{1}\right\rangle\left|R^{\prime}, R^{\prime}\right\rangle+\left|\psi_{2}\right\rangle\left|R^{\prime}, R^{\prime}\right\rangle\right],
$$

where $|L, L\rangle$ are undeflected left-handed entangled photon states and $\left|R^{\prime}, R^{\prime}\right\rangle$ are deflected right-handed entangled photon states. This expression combines the various elements of the system (i.e., double-slit screen, PPS filters, and entangled incident photons) in a non-separable [18] manner which explains the observed correlations.

The probability density $P_{B}(\boldsymbol{R})$ for $\gamma_{B}$ arriving at a point $\boldsymbol{r}=\boldsymbol{R}$ on the observation screen is given by the squared modulus of $\Psi_{B}(R)$, that is

$$
P_{B}(\boldsymbol{R})=\left|\langle\boldsymbol{R} \mid \Psi\rangle_{B}\right|^{2}=\frac{1}{2}\left[\left|\psi_{1}\right|^{2}+\left|\psi_{2}\right|^{2}+\left\langle\psi_{1} \mid \psi_{2}\right\rangle+\left\langle\psi_{2} \mid \psi_{1}\right\rangle\right],
$$

where the cross-terms $\left\langle\psi_{1} \mid \psi_{2}\right\rangle$ and $\left\langle\psi_{2} \mid \psi_{1}\right\rangle$ are responsible for the well-known interference phenomena of Young's double-slit experiment. This result, of course, agrees with our expectations that the two-slit interference apparatus with parallel PPS filters should behave similar to that without filters and produce an interference pattern on the observation screen. This result also ensures that the PPS filters are operating properly and not causing the incident photons to experience any large uncontrollable phase factors and or scattering events before passing through the screen.

\section{Observing Position without Destroying Interference}

Now we consider the situation where the PPS filters positioned in front of the slits are oriented so that left-handed photons can pass through hole 1 without divergence and right-handed photons can pass through hole 2 without divergence. The incident photons that pass through the antiparallel PPS filters are transmitted without disturbing the coherent superposition of $\left|\psi_{1}\right\rangle$ and $\left|\psi_{2}\right\rangle$. For this set up, the state of the photons $\gamma_{B}$ exiting the double-slit screen is given by

$$
|\Psi\rangle_{B}=\frac{1}{2}\left[\left|\psi_{1}\right\rangle|L, L\rangle+\left|\psi_{2}\right\rangle|R, R\rangle+\left|\psi_{1}\right\rangle\left|R^{\prime}, R^{\prime}\right\rangle+\left|\psi_{2}\right\rangle\left|L^{\prime}, L^{\prime}\right\rangle\right]
$$

Because the PPS filters do not alter the polarization state of the incident photons in any way, the superposition of the two slits remains intact. Hence, as expressed by Equation (5) the deflected states $\left|\psi_{1}\right\rangle\left|R^{\prime}, R^{\prime}\right\rangle$ and $\left|\psi_{2}\right\rangle\left|L^{\prime}, L^{\prime}\right\rangle$ do not vanish. In general, if the path length difference within the filter for the deflected photons is made to be an integral number of wavelengths of the undeflected incident photons, then it follows: 


$$
\left\langle R, R \mid R^{\prime}, R^{\prime}\right\rangle=1 \text { and }\left\langle R, R \mid L^{\prime}, L^{\prime}\right\rangle=0 .
$$

From this it follows that the probability density $P_{B}(\boldsymbol{R})$ for $\gamma_{B}$ arriving at a point $\boldsymbol{r}=\boldsymbol{R}$ on the observation screen is given by

$$
P_{B}(\boldsymbol{R})=\left|\langle\boldsymbol{R} \mid \Psi\rangle_{B}\right|^{2}=\frac{1}{2}\left[\left|\psi_{1}\right|^{2}+\left|\psi_{2}\right|^{2}+\left\langle\psi_{1} \mid \psi_{2}\right\rangle+\left\langle\psi_{2} \mid \psi_{1}\right\rangle\right]
$$

and the cross-terms $\left\langle\psi_{1} \mid \psi_{2}\right\rangle$ and $\left\langle\psi_{2} \mid \psi_{1}\right\rangle$ responsible for the interference phenomena remain. This result agrees with Equation (4).

In the setup shown in Figure 3 the spin of the non-incident photons is measured by a spin photodetector. Measurement of the spin orientation of $\gamma_{A}$ before $\gamma_{B}$ arrives at the observation screen or after the following entangled photon enters the apparatus [19], is observed to collapse the coherent superpositioned probability waves of the entangled photons that freely pass through the screen; i.e., $\left|\psi_{1}\right\rangle\left|R^{\prime}, R^{\prime}\right\rangle=\left|\psi_{2}\right\rangle\left|L^{\prime}, L^{\prime}\right\rangle=0$, which causes the interference terms of the probability density $P_{B}(\boldsymbol{R})$ to vanish as well. However, by performing an intermediate delayed choice (IDC) measurement of the spin of $\gamma_{A}$ after $\gamma_{B}$ has arrived at the observation screen $\sigma$ leaves the interference pattern intact as displayed in Figure 3. Note that an IDC measurement is defined here as the measurement of the spin orientation of an entangled photon after it has arrived at the observation screen for one photon pair in (quantum) superposition with the two-slit interference apparatus at a time. Thus, an IDC measurement of $\gamma_{A}$ allows us to indirectly determine which slit $\gamma_{B}$ went through without collapsing the wavefunction of its coherent superpositioned state vector $|\Psi\rangle_{B}$, and thereby allow interference fringes to appear on the observation screen undisturbed.

For example, assume an IDC measurement of $\gamma_{A}$ gives a right-handed outcome, then it immediately follows from the conservation of total spin angular momentum, that $\gamma_{B}$ had the same spin when it passed through the screen $\Sigma$. Since the PPS filters, located in front of the two narrow slits, prevents photons with right-handed spin from arriving at the observation screen through slit 1

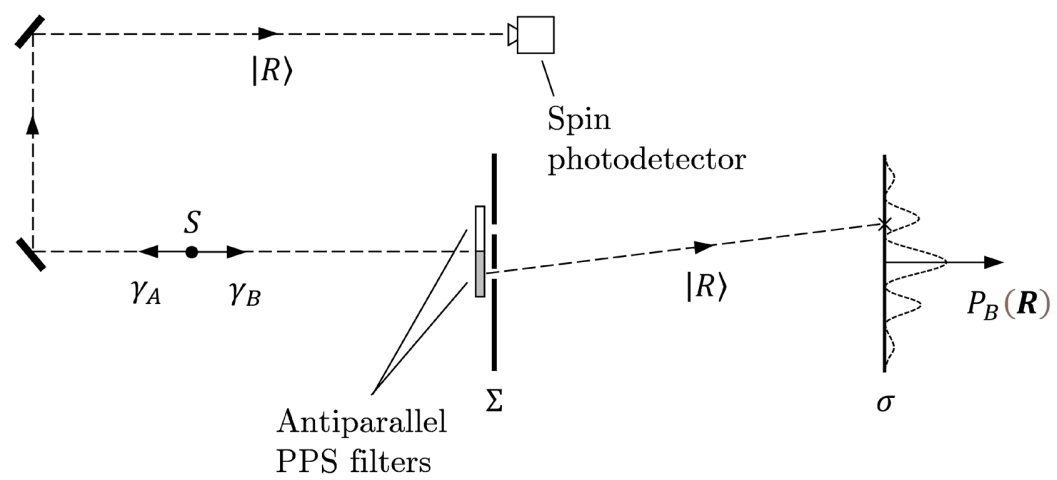

Figure 3. Photon $\gamma_{B}$ of an entangled photon pair, emitted by a spin-zero light source $S$, is incident on a screen $\Sigma$ with two narrow slits covered by two antiparallel PPS filters. An IDC measurement of $\gamma_{A}$ by a spin photodetector leaves the interference pattern, on the observation screen $\sigma$, undisturbed. 
and photons with left-handed spin from arriving at the observation screen through slit 2, then we can logically deduce with a high degree of confidence that $\gamma_{B}$ must have passed through slit 2 to reach the observation screen $\sigma$.

\section{Conclusions}

We have thus presented a compelling thought experiment that allows us to observe the position of a photon in Young's two-slit apparatus without causing the interference pattern to vanish. In general, the advanced optical hardware and measurement techniques presented in this paper are considered to make this thought experiment notably different than similar experiments [19] [20] of its kind.

In any event, the implications of this thought experiment are immense. On the one hand, observation of the position of a particle while behaving like a wave would fundamentally alter our physical understanding of the wave-particle duality of nature and perhaps provide new insight into the inner workings of quantum physics as well. While on the other hand, if the antiparallel PPS filters do not destroy the interference pattern and the position of the photon still eludes us, then this result would strongly suggest that the information of which slit the photon passed through to produce an interference pattern is causally (temporally) inaccessible.

\section{Conflicts of Interest}

The author declares no conflicts of interest regarding the publication of this paper.

\section{References}

[1] Heisenberg, W. (1925) Uber quantentheoretische Umdeutung kinematischer und mechanischer Beziehungen. Zeitschrift für Physik, 33, 879-893. https://doi.org/10.1007/BF01328377

[2] Born, M. and Jordan, P. (1925) On Quantum Mechanics. Zeitschrift für Physik, 34, 858-888. https://doi.org/10.1007/BF01328531

[3] Schrodinger, E. (1926) Quantisierung als Eigenwertproblem. Annalen der Physik, 384, 361-376. https://doi.org/10.1002/andp.19263840404

[4] de Broglie, L. (1925) Recherches sur la théorie des quanta. Annals of Physics, 10, 22-128. https://doi.org/10.1051/anphys/192510030022

[5] de Broglie, L. (1929) The Wave Nature of the Electron. Nobel Lecture.

[6] Young, T. (1807) A Course of Lectures on Natural Philosophy and the Mechanical Arts. Printed for J. Johnson, London. https://doi.org/10.5962/bhl.title.22458

[7] Ke, Y., Liu, Z., Liu, Y., Zhou, J., Shu, W., Luo, H. and Wen, S. (2016) Compact Photonic Spin Filters. Applied Physics Letters, 109, Article ID: 181104. https://doi.org/10.1063/1.4966954

[8] Peng, J., Cumming, B. and Gu, M. (2019) Direct Detection of Photon Spin Angular Momentum by a Chiral Graphene Mid-Infrared Photodetector. Optics Letters, 44, 2998-3001. https://doi.org/10.1364/OL.44.002998

[9] Einstein, A., Podolsky, B. and Rosen, N. (1935) Can Quantum-Mechanical Descrip- 
tion of Physical Reality Be Considered Complete? Physical Review, 47, 777-780. https://doi.org/10.1103/PhysRev.47.777

[10] Bohm, D. (1951) Quantum Theory. Prentice-Hall, Englewood Cliffs.

[11] Allen, L., Barnett, S. and Padgett, M. (2020) Optical Angular Momentum, 1st Edition, CRC Press, Boca Raton.

[12] Taylor, G.I. (1909) Interference Fringes with Feeble Light. Proceedings of the Cambridge Philosophical Society, 15, 114-115.

[13] Dirac, P.A.M. (1958) The Principles of Quantum Mechanics, 4th Edition, Oxford University Press, Oxford.

[14] Schrödinger, E. (1935) Die gegenwärtige Situation in der Quantenmechanik (The Present Situation in Quantum Mechanics). Naturwissenschaften, 23, 807-812. https://doi.org/10.1007/BF01491891

[15] Feynman, R., Leighton, R. and Sands, M. (1963) The Feynman Lectures on Physics. Vol. III, Addison-Wesley Pub. Co., Reading.

[16] Fox, M. (2006) Quantum Optics: An Introduction. Oxford University Press, New York.

[17] Shih, Y.H. and Alley, C.O. (1988) New Type of Einstein-Podolsky-Rosen-Bohm Experiment Using Pairs of Light Quanta Produced by Optical Parametric Down Conversion. Physical Review Letters, 61, 2921-2924. https://doi.org/10.1103/PhysRevLett.61.2921

[18] Costa, G. (2003) Entanglement and Non-Separability in Quantum Mechanics. In: Di Bartolo, B., Ed., Spectroscopy of Systems with Spatially Confined Structures, Vol. 90, Springer, Dordrecht, 593-306. https://doi.org/10.1007/978-94-010-0287-5 18

[19] Walborn, S.P., Cunha, T.M.O., Pádua, S. and Monken, C.H. (2002) Double-Slit Quantum Eraser. Physical Review A, 65, Article ID: 033818. https://doi.org/10.1103/PhysRevA.65.033818

[20] Scully, M., Englert, B. and Walther, H. (1991) Quantum Optical Tests of Complementarity. Nature, 351, 111-116. https://doi.org/10.1038/351111a0 